\title{
GROUP ACTIONS ON HOMOLOGY QUATERNIONIC PROJECTIVE PLANES
}

\author{
STEVEN H. WEINTRAUB
}

\begin{abstract}
A class of $\mathbf{Z}_{p}$-actions, resembling well-known actions on the quaternionic projective plane, is defined and studied. The existence of such actions on a closed homology quaternionic projective plane is shown to imply numerical restrictions on the manifold's Pontrjagin classes. One consequence is that for $p=3$, or 5 , infinitely many smooth manifolds of this type admit no smooth $\mathbf{Z}_{\boldsymbol{p}}$-actions.
\end{abstract}

In this paper, we consider the question of the existence of locally smooth cyclic group actions on simply-connected 8-manifolds having the same homology as $\mathbf{H} P^{2}$, the quaternionic projective plane. Such manifolds have a very simple cell structure, and they have been classified (see $\$ 1$ ).

We are in particular concerned with $\mathbf{Z}_{p}$-actions, for $p$ an odd prime. From Smith theory, such an action must have a nonempty fixed-point set. We prove nonexistence results by applying the $G$-signature theorem to the normal bundle of the fixed point set, and we construct actions by equivariantly regluing bundles.

Some of our results here are special cases of results in [7], but in the specific case considered here we obtain much stronger information than in general. Also, it is interesting to compare Theorem 3.1 of this paper, which roughly

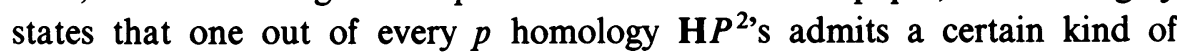
smooth $\mathbf{Z}_{p}$-action, with [7, Corollary 3.8] which shows that every homology $\mathbf{H} \boldsymbol{P}^{2}$ admits many PL $\mathbf{Z}_{p}$-actions.

The author would like to thank Reinhard Schultz for several valuable suggestions, and in particular for strengthening Theorem 3.1.

\section{Preliminaries.}

Notation. Throughout this paper, $V$ denotes a 1-connected (integral) homology $\mathbf{H} P^{2}, \xi \in H^{4}(V)$ a generator. $F \subset V$ is the fixed-point set of a $\mathbf{Z}_{p}$-action, and $p$ is an odd prime.

Eells and Kuiper have classified homology $\mathbf{H} P^{2}$ 's as follows:

THEOREM [5]. Let $V$ be as above; then for some integer $h$, we have

$$
p_{1}(V)=2(2 h-1) \xi, \quad p_{2}(V)=\left(\left[45+4(2 h-1)^{2}\right] / 7\right) \xi^{2}
$$

Received by the editors June 23, 1977 and, in revised form, October 3, 1977. AMS (MOS) subject classifications (1970). Primary 57E10, 57E15, 57E25; Secondary 57D55. Key words and phrases. Cyclic group actions, quaternionic projective plane. 
Furthermore, if we write $V=V_{h}$ where $p_{1}(V)=2(2 h-1) \xi$, the following hold:

(1) $h$ determines $V_{h}$ up to orientation preserving $P L$ homeomorphism, and $V_{h}$ is $P L$ homeomorphic to $-V_{1-h}$.

(2) The oriented homotopy type of $V$ is determined by $h \bmod 12$.

(3) $V_{1}=\mathbf{H} P^{2}, V_{0}=-\mathbf{H} P^{2}$.

(4) $V_{h}$ is smoothable iff $h \equiv 0,1,8,49$ (56), and is a smooth structure on a homotopy $\mathbf{H} P^{2}$ iff $h \equiv 0,1,49,120$ (168).

(5) If $V_{h}$ is smooth, $h$ determines its diffeomorphism type up to the action of $\Theta_{8}=Z_{2}$. Whether this action is effective is unknown.

If $V$ is a homology $\mathbf{H} P^{2}$ with $\mathrm{Z}_{p}$-action, the following theorem of Bredon [2, Theorem VII.3.1] describes its fixed point set $F$.

THEOREM. $H^{*}\left(F: \mathbf{Z}_{p}\right)$ is one of the following possibilities: A. $H^{*}\left(S^{4} \cup\right.$ pt : $\left.\mathbf{Z}_{p}\right)$, B. $H^{*}\left(\mathbf{C} P^{2}: \mathbf{Z}_{p}\right)$, C. $H^{*}\left(S^{2} \cup \mathrm{pt}: \mathbf{Z}_{p}\right)$, D. $H^{*}\left(3 \mathrm{pts}: \mathbf{Z}_{p}\right)$.

If $V$ is a homotopy $\mathbf{H} P^{2}$ case $\mathrm{D}$ cannot occur for $p=3$. In case $\mathrm{A}$, $H^{4}\left(V: \mathbf{Z}_{p}\right) \rightarrow H^{4}\left(F: \mathbf{Z}_{p}\right)$ is an isomorphism.

(Note that a 1-connected $\mathrm{Z}_{p}$-homology $S^{4}$ is homotopy-equivalent to $S^{4}$, and similarly for $\mathbf{C} P^{2}$.)

The above possibilities are easily realized as fixed-point sets. Write $\mathbf{H} P^{2}$ as the right quaternionic projective space, i.e.

$$
\mathbf{H} P^{2}=\left\{\left(z_{0}, z_{1}, z_{2}\right) \in \mathbf{H}^{3}-(0,0,0) \mid\left(z_{0}, z_{1}, z_{2}\right)=\left(z_{0} \lambda, z_{1} \lambda, z_{2} \lambda\right), \lambda \in \mathbf{H}\right\} .
$$

Then there are linear actions of $\mathbf{Z}_{p}$ on $\mathbf{H} P^{2}$ given by

$$
t\left[z_{0}, z_{1}, z_{2}\right]=\left[t^{a_{0}} z_{0}, t^{a_{1}} z_{1}, t^{a_{2}} z_{2}\right], \quad t=\exp (2 \pi i / p),
$$

and the following result is a routine verification (compare [2, Chapter VII]):

Proposition A. If $a_{2} \neq a_{0}=a_{1}=0, F=S^{4} \cup \mathrm{pt}$ with normal representations $\left(2 a_{2}, 2 a_{2}\right)$ at $S^{4},\left(2 a_{2}, 2 a_{2}, 2 a_{2}, 2 a_{2}\right)$ at the isolated point.

B. If $\left|a_{0}\right|=\left|a_{1}\right|=\left|a_{2}\right| \neq 0, F=\mathbf{C} P^{2}$ with normal representation $\left(2 a_{0}, 2 a_{0}\right)$.

C. If $0 \neq\left|a_{0}\right|=\left|a_{1}\right| \neq\left|a_{2}\right|, F=S^{2} \cup$ pt with normal representation $\left(a_{0}, a_{0}\right.$ $\left.-a_{2}, a_{0}+a_{2}\right)$ at $S^{2},\left(a_{0}-a_{2}, a_{0}+a_{2}, a_{0}-a_{2}, a_{0}+a_{2}\right)$ at the isolated point.

D. If $\left|a_{0}\right| \neq\left|a_{1}\right| \neq\left|a_{2}\right| \neq\left|a_{0}\right|, F=3$ points with normal representation ( $a_{0}$ $\left.a_{1}, a_{0}+a_{1}, a_{0}-a_{2}, a_{0}+a_{2}\right)$ at $[1,0,0]$ and similarly for $[0,1,0]$ and $[0,0,1]$.

In the above, the symbol (a) means that $t$ acts on a subspace of a normal slice to $F$ by $z \rightarrow t^{a} \cdot z$. Note that the integers (a) are only determined up to sign.

Definition 1.1. If $\mathbf{Z}_{p}$ acts locally smoothly and the normal representations of $\mathbf{Z}_{p}$ at $F$ are the same as those for a linear action, we shall call the action quasi-linear (compare [4]).

Definition 1.2. If $f: M^{4} \rightarrow V$ with $\left\langle f\left(\left[M^{4}\right]\right), \xi\right\rangle=d$, we will say that $f$ is of degree $d$.

\section{High-dimensional fixed-point sets.}

LEMMA 2.1. If $S^{4} \rightarrow V$ is an embedding of degree $d$, then $\chi\left(\nu\left(S^{4}\right)\right)=d^{2}$. 
Proof. If $d=0, S^{4}$ is inessential and $\nu$ is trivial, so $\chi(\nu)=0$. If $d \neq 0$, consider the exact sequence of the pair $\left(V, S^{4}\right)$ :

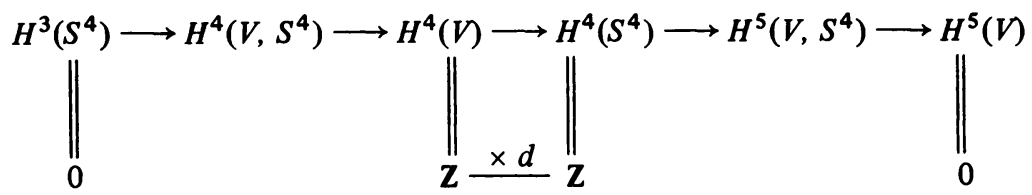

Hence $H^{4}\left(V, s^{4}\right)=0=H^{4}\left(V-S^{4}\right), H^{5}\left(V, S^{4}\right)=\mathbf{Z}_{d}=H_{3}\left(V-S^{4}\right)$ by Alexander duality.

Now consider the Mayer-Vietoris sequence for $\left(V, E\left(\nu\left(S^{4}\right)\right)\right.$, $\left.\overline{V-E\left(\nu\left(S^{4}\right)\right)}\right)$ :

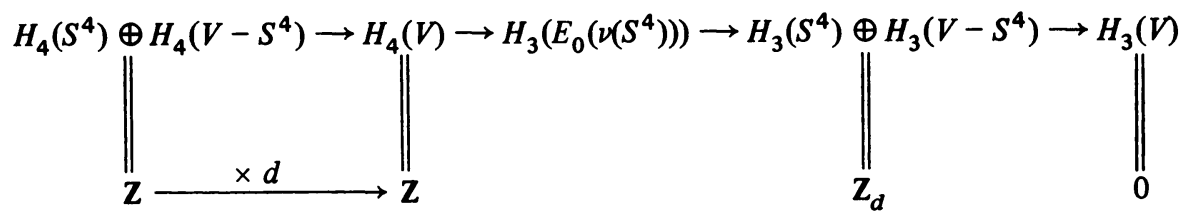

Hence $H^{4}\left(E_{0}\left(\nu\left(S^{4}\right)\right)\right)=H_{3}\left(E_{0}\left(\nu\left(S^{4}\right)\right)\right)=\mathbf{Z}_{d^{2}}$ or $\left(\mathbf{Z}_{d}\right)^{2}$. But we have the Gysin sequence:

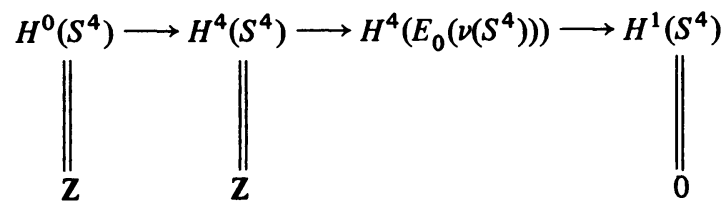

so $H^{4}\left(E_{0}\left(\nu\left(S^{4}\right)\right)\right)$ is cyclic, so equal to $\mathbf{Z}_{d^{2}}$, so $\chi\left(\nu\left(S^{4}\right)\right)=d^{2}$.

THEOREM 2.2. Let $\mathbf{Z}_{p}$ act locally smoothly on $V$ with $H^{*}\left(F: \mathbf{Z}_{p}\right)=H^{*}\left(S^{4} \cup\right.$ $\left.\mathrm{pt}: \mathbf{Z}_{p}\right)$. Then $V$ is almost diffeomorphic to $\mathbf{H} P^{2}$ and the action is quasi-linear. Furthermore, the inclusion $F^{\prime} \rightarrow V, F^{\prime}$ the 4-dimensional component of $F$, has degree \pm 1 .

Proof. First we show that if the normal representation at $F^{\prime}$ is $t\left(z_{0}, z_{1}\right)=$ $\left(e^{i \theta} z_{0}, e^{i \theta^{\prime}} z_{1}\right), 0<\theta<\pi, 0<\theta^{\prime}<\pi$, then $\theta=\theta^{\prime}$. For if $\theta \neq \theta^{\prime}, \nu\left(F^{\prime}\right)$ splits into a sum of two complex line bundles, and hence is of finite order in $K\left(F^{\prime}\right)$, as $H^{2}\left(F^{\prime}: \mathbf{Q}\right)=0$.

However, Bredon's theorem states that $d$, the degree of the inclusion of $F^{\prime}$, is prime to $p$, and hence nonzero, so $c_{2}(\nu)=\chi(\nu)=d^{2}\left\{F^{\prime}\right\} \in H^{4}\left(F^{\prime}: \mathbf{Q}\right)$ is a class of infinite order, which is a contradiction. Hence $\theta=\theta^{\prime}$.

Now suppose the normal representation at the isolated point of $t$ is $e^{i \theta_{1}} \oplus e^{i \theta_{2}} \oplus e^{i \theta_{3}} \oplus e^{i \theta_{4}}, 0<\theta_{i}<\pi$. We must show $\theta_{1}=\theta_{2}=\theta_{3}=\theta_{4}=\theta$. Apply the Atiyah-Singer $G$-signature formula to this action (assume $V$ is oriented so that its intersection form is (1) rather than $(-1)$, for the sake of definiteness) (see [1, p. 589]). Then 


$$
\begin{aligned}
1= & \prod_{i=1}^{4} \cot \theta_{i} / 2 \\
& +\left\{4 \cdot\left[-\cot ^{2} \theta / 2\right] \cdot\left[\frac{-c_{2}}{4 \sin ^{2} \theta / 2 \cos ^{2} \theta / 2}-\frac{c_{1}^{2}-2 c_{2}}{4 \sin ^{2} \theta / 2}\right]\right\}\left[F^{\prime}\right] . \\
c_{1}= & 0 \text { as } H^{2}\left(F^{\prime}: \mathbf{Q}\right)=0 . \text { Write } c_{2} \text { for }\left\langle c_{2},\left[F^{\prime}\right]\right\rangle \in \mathbf{Q} . \\
1= & \prod_{i=1}^{4} \cot \theta_{i} / 2+\left\{\frac{\cos ^{2} \theta / 2}{\sin ^{2} \theta / 2}\left[\frac{c_{2}}{\sin ^{2} \theta / 2 \cos ^{2} \theta / 2}-\frac{2 c_{2}}{4 \sin ^{2} \theta / 2}\right]\right\} \\
= & \prod_{i=1}^{4} \cot \theta_{i} / 2+c_{2}\left(\left[1-2 \cos ^{2} \theta / 2\right] / \sin ^{4} \theta / 2\right) .
\end{aligned}
$$

Now the linear action on $\mathbf{H} P^{2}$ has $c_{2}=1, \theta_{i}=\theta, i=1, \ldots, 4$, giving

$$
1=\cot ^{4} \theta / 2+\left(\left[1-2 \cos ^{2} \theta / 2\right] / \sin ^{4} \theta / 2\right) \text {. }
$$

Subtracting,

or

$$
\begin{aligned}
\prod_{i=1}^{4} \cot \theta_{i} / 2 & =\cot ^{4} \theta / 2+\left(1-c_{2}\right)\left[\frac{1-2 \cot ^{2} \theta / 2}{\sin ^{4} \theta / 2}\right] \\
& =\cot ^{4} \theta / 2+\left(1-c_{2}\right) \cot ^{4} \theta / 2\left[\frac{1-2 \cos ^{2} \theta / 2}{\cos ^{4} \theta / 2}\right]
\end{aligned}
$$

$$
\prod_{i=1}^{4} \frac{\cot \theta_{i} / 2}{\cot \theta / 2}=1+\left(1-c_{2}\right)\left[\tan ^{4} \theta / 2-1\right]
$$

Now $0<\theta<\pi, 0<\theta_{i}<\pi$, $e^{i \theta}$ is a $p$ th root of unity. If $p=3, \theta=\theta_{1}$ $=\cdots=\theta_{4}=2 \pi / 3$ so the action is of quasi-linear type. If $p \neq 3$, choose $t$ so that $\theta=2 \pi / p$. Then as $p \neq 3,\left|\tan ^{4} \theta / 2\right|<\frac{1}{2}$. Also, $\left|\cot \theta_{i} / 2\right|<|\cot \theta / 2|$ with equality only for $\theta_{i}=\theta$. Therefore, the left-hand side of (*) lies between 0 and 1 , so we must have $c_{2}=0$ or 1 . But $c_{2}=\chi\left(\nu\left(F^{\prime}\right)\right)=d^{2} \neq 0$ (where $d$ is the degree of $F^{\prime} \rightarrow V$ ). Hence $c_{2}=1, d= \pm 1$, and $\theta_{i}=\theta \forall i$. Finally, as $f^{\prime}$ has degree $+1, p_{1}(V)=2 c_{2}\left(\nu\left(F^{\prime}\right)\right)=2$, so $V$ is almost diffeomorphic to $\mathbf{H} \boldsymbol{P}^{2}$.

THEOREM 2.3. Let $\mathbf{Z}_{p}$ act locally smoothly on $V$ with $H^{*}\left(F: \mathbf{Z}_{p}\right)=$ $H^{*}\left(\mathbf{C P}^{2}: \mathbf{Z}_{p}\right)$. Then $V$ is almost diffeomorphic to $\mathbf{H} P^{2}$ and the action is quasi-linear. Also, the inclusion $F \rightarrow V$ has degree \pm 1 .

Proof. Suppose the normal representation is given by $t \rightarrow e^{i \theta_{0}} \oplus e^{i \theta_{1}}$, $0<\theta_{i}<\pi$. We must show $\theta_{0}=\theta_{1}$. If not, $\nu(F)$ splits into a sum of complex line bundles $\nu=\eta_{0} \oplus \eta_{1}$. From the proof of Lemma 1, we see that if $F \rightarrow V$ is of degree $d$,

$$
\chi(\nu(F))=d^{2}=c_{2}(\nu(F))=c_{2}\left(\eta_{0} \oplus \eta_{1}\right)=c_{1}\left(\eta_{0}\right) c_{1}\left(\eta_{1}\right) \in H^{4}(F: \mathbf{Q}) .
$$

Now consider the restrictions of $\eta_{1}$ and $\eta_{2}$ to $F^{2}$, the 2-skeleton of $F$. As $V$ is 3-connected, $\tau(V) \mid F^{2}$ is trivial, so 


$$
\begin{aligned}
0 & =c_{1}\left(\tau(V) \mid F^{2}\right)=c_{1}\left(\tau(F)\left|F^{2} \oplus \nu(F)\right| F^{2}\right) \\
& =c_{1}\left(\tau(F)\left|F^{2} \oplus \eta_{0}\right| F^{2} \oplus \eta_{1} \mid F^{2}\right)=3+c_{1}\left(\eta_{0}\right)+c_{1}\left(\eta_{1}\right) .
\end{aligned}
$$

Thus we have the system of equations

$$
c_{1}\left(\eta_{0}\right) \cdot c_{1}\left(\eta_{1}\right)=d^{2}, \quad c_{1}\left(\eta_{0}\right)+c_{1}\left(\eta_{1}\right)=-3,
$$

whose only solution in integers is $d=0, c_{1}\left(\eta_{i}\right)=-3, c_{1}\left(\eta_{1-i}\right)=0$.

Suppose that $c_{1}\left(\eta_{0}\right)=0$. Then $\eta_{0}$ is of finite order in $K(F)$. As $d=0, F$ is contractible in $V$, so $\nu(F)$ in $V$ is the abstract normal bundle of $F$. Thus, $\eta_{1}$ is a line bundle with the same rational Pontrjagin classes as $\nu(F)$, so $-3=$ $p_{1}\left(\eta_{1}\right)=\chi\left(\eta_{1}\right)^{2}$ which is impossible, so $\theta_{0}=\theta_{1}$.

Now we apply the Atiyah-Singer formula to this action. $\chi(\nu(F))=c_{2}(\nu(F))$ $=d^{2}$. Also, $p_{1}(\tau(F))=3 I(F)=3$ from the Hirzebruch formula. If $p_{1}(V)=$ $2(2 h-1)$, then as $\nu(F) \oplus \tau(F)=\tau(V) \mid F, p_{1}(\nu(F))=2(2 h-1) d-3=\left(c_{1}^{2}\right.$ $\left.-2 c_{2}\right)(\nu(F))$. If $\{F\}$ is dual to $[F]$, we have

$$
\begin{aligned}
1 & =\left(-2^{2} \cot ^{2} \theta / 2\right) \\
& \cdot\left\{[ 1 + \{ F \} / 4 ] \cdot \left[1-\frac{d^{2}\{F\}}{4 \sin ^{2} \theta / 2 \cos ^{2} \theta / 2}\right.\right.
\end{aligned}
$$

$$
\left.\left.-\frac{(2(2 h-1) d-3)\{F\}}{4 \sin ^{2} \theta / 2}\right]\right\}[F] .
$$

The linear action yields the equation:

$$
\begin{aligned}
1= & \left(-2^{2} \cot ^{2} \theta / 2\right) \\
& \cdot\left\{[1+\{F\} / 4]\left[1-\frac{\{F\}}{4 \sin ^{2} \theta / 2 \cos ^{2} \theta / 2}+\frac{\{F\}}{4 \sin ^{2} \theta / 2}\right]\right\}[F] .
\end{aligned}
$$

Subtracting,

$$
0=\left(d^{2}-1\right)+(2(2 h-1) d-2) \cos ^{2} \theta / 2 .
$$

As for $p>3, \cos ^{2} \theta / 2$ is irrational, the right side must vanish identically, yielding $h=1, d=1$ or $h=0, d=-1$ corresponding to the two orientations of $\mathbf{H} P^{2}$.

For $p=3$ we obtain a quadratic equation whose discriminant is $(2 h-1)^{2}$ +12 , which is never a perfect square, so in this case also (*) must vanish identically.

REMARK 2.4. If $\mathbf{Z}_{p}$ acts smoothly on $V_{h}, p$ is odd, and $\Sigma^{8}$ is a homotopy sphere, then $\mathbf{Z}_{p}$ acts smoothly on $V_{h} \# \Sigma^{8}$ even if the latter is not diffeomorphic to $V_{h}$. A standard cutting and pasting trick in [6] implies that $V_{h} \# p \Sigma^{8}$ admits a smooth $\mathbf{Z}_{p}$-action, and $\theta_{8}=\mathbf{Z}_{2}$ implies $p \Sigma=\Sigma$.

3. Low dimensional fixed-point sets. We now consider the cases where $F=S^{2} \cup$ pt or 3 points.

THEOREM 3.1. Let $V$ be a 1-connected homology $\mathbf{H} P^{2}$. Then $V$ admits $a$ 
quasi-linear, locally smooth $\mathbf{Z}_{p}$-action with fixed-point set $F=S^{2} \cup \mathrm{pt}$ or, for $p \neq 3,3$ isolated points, if and only if $\left\langle p_{1}(V) \cup \xi,[V]\right\rangle= \pm 2(4 p)$.

Proof. Consider linear actions of $\mathbf{Z}_{p}$ on $\pm \mathbf{H} P^{2}$ (with $\left\langle p_{1}\left( \pm \mathbf{H} P^{2}\right) \cup \xi\right.$, $\left.\left[ \pm \mathbf{H} P^{2}\right]\right\rangle= \pm 2$ ) with fixed-point set as indicated. Each such action has an invariant 4-sphere $S^{4}$, containing all but one of the fixed points, representing the homology class dual to $\xi$. In fact, $p_{1}\left( \pm \mathbf{H} P^{2}\right)=i_{*} p_{1}\left(\nu\left(S^{4}\right)\right)$, where $i$ denotes the inclusion and $\nu\left(S^{4}\right)$ the normal bundle.

If $F=3$ points, we can construct the actions for the "if" half of the theorem as follows: Since the invariant $S^{4}$ in a linear action contains two fixed points, $\mathbf{Z}_{p}$ acts freely on an invariant equator $S^{3}$. The normal bundle of $S^{4}$ is then determined by a $Z_{p}$-equivariant map $\nu_{0}^{\#}: S^{3} \rightarrow O_{4}$, and the desired examples will be constructed to have other such equivariant normal bundles, by choosing different equivariant maps $\nu^{\#}: S^{3} \rightarrow O_{4}$. Then $\nu^{\#}$ must differ from $\nu_{0}^{\#}$ in $\pi_{3}\left(O_{4}\right)$ by a map $\tilde{\alpha}: S^{3} \rightarrow O_{4}$ which factors through the covering map $S^{3} \stackrel{\pi}{\rightarrow} L^{3}(p)$. By obstruction theory any map of $L^{3}(p)$ into $O_{4}$ must factor through the collapse $L^{3}(p) \stackrel{c}{\rightarrow} S^{3}$, so $\tilde{\alpha}$ must be of the form $S^{3} \stackrel{\pi}{\rightarrow} L^{3}(p) \stackrel{c}{\rightarrow} S^{3} \stackrel{\alpha}{\rightarrow} O_{4}$, where $\pi$ has degree $p, c$ has degree 1 , and $\alpha$ is arbitrary. However, as the bundles determined by $\nu^{\#}$ and $\nu_{0}^{\#}$ must have the same Euler class, $\alpha$ must in fact factor through the inclusion of $O_{3}$ into $O_{4}$. If we choose $\alpha=k$ times a generator of $\pi_{3}\left(O_{3}\right) \subseteq \pi_{3}\left(O_{4}\right)$, we get a new equivariant map $v_{k}$ with associated closed tube $T_{k}\left(S^{4}\right)$, and it is easy to check that the Pontrjagin classes of $v_{k}^{\#}$ and $\nu_{0}^{\#}$ differ by $4 k p$.

The boundary of $T_{k}\left(S^{4}\right)$ is a smooth homotopy 7 -sphere with free $\mathbf{Z}_{p}$ action. We claim $\partial T_{k}\left(S^{4}\right)$ is equivariantly PL equivalent to the linear action on $S^{7}$ given by the linear model; by the work of Browder, Petrie, and Wall [3], it suffices to show these actions have the same Atiyah-Singer and Reidemeister torsion invariants.

The Atiyah-Singer invariants are clearly equal since we have changed neither the intersection form in the tube nor the action in a neighborhood of the fixed points, and the Reidemeister torsion invariants are equal because $\partial T_{k}$ and $S^{7}$ are both obtained by gluing the same two PL (in fact smooth) manifolds $M_{1}$ and $M_{2}$ together by a PL homeomorphism (in fact diffeomorphism) of their boundaries. Therefore $\partial T_{k}\left(S^{4}\right)$ is equivariantly PL equivalent with the linear $S^{7}$ in the linear model and $V\langle k\rangle=T_{k}\left(S^{4}\right) \cup$ cone $\partial T_{k}\left(S^{4}\right)$ is a $\mathbf{Z}_{p}$-quasilinear homology $\mathbf{H} P^{2}$. The final sentence of the previous paragraph implies that the Pontrjagin classes of $V\langle k\rangle$ and $\mathbf{H} P^{2}$ differ by $4 k p$.

Conversely, if $F=3$ points we show in [7, Lemma 2.3] that any such $\mathrm{Z}_{p}$-action on $V$ contains a degree 1 invariantly immersed $S^{4}$ containing two fixed points, so by the argument above, $\left\langle p_{1}( \pm V) \cup \xi,[ \pm V]\right\rangle$ must differ from $\left\langle p_{1}\left( \pm \mathbf{H} P^{2}\right) \cup \xi,\left[ \pm \mathbf{H} P^{2}\right]\right\rangle$ by a multiple of $4 p$.

In case $F=S^{2} \cup \mathrm{pt}$, the "if" half is entirely similar, and as for the "only if" half, an obstruction theory argument shows that the inclusion of the fixed $S^{2}$ in $V / \mathbf{Z}_{p}$ can be extended to a map of $S^{4} / \mathbf{Z}_{p}$ into $V / \mathbf{Z}_{p}$, yielding a degree 
1 map, whose cover may be deformed into an immersion of $S^{4}$ into $V$, and the argument then proceeds as above.

Let us take this opportunity to correct an oversight in [7, §3] where we neglected to observe the analogous condition on $\alpha$ (same notation!) as above-that it not change the Euler class. Thus we must change the definition of $b_{k}$ in [7] to be the minimum Pontrjagin class of an $O_{2 k-1}$-bundle over $S^{2 k}$.

COROLlaRY 3.2. If $V$ is smooth and $p \neq 7$, the actions constructed in Theorem 3.1 are smooth.

Proof. We have shown that $\partial T_{k} / Z_{p}$ is $\mathrm{PL}$ homeomorphic to $L^{7}(p)$; we need only show further that it is diffeomorphic. Now $\partial T_{k} / \mathbf{Z}_{p}$ must be diffeomorphic to $L^{7}(p) \# \Sigma^{7}$ for some homotopy sphere $\Sigma^{7}$, so $\partial T_{k}$ must be diffeomorphic to $S^{7} \# p \Sigma^{7}=p \Sigma^{7}$. But if $V_{k}$ is smooth, $\partial T_{k}$ must be diffeomorphic to $S^{7}$, so $p \Sigma^{7}=S^{7}$, and hence as $\theta_{7}$ has order 28 , for $p \neq 7$, $\Sigma^{7}=S^{7}$, and so the action is smooth.

Note that by Remark 2.4, the smooth actions constructed on $V$ above also yield smooth actions on $V \# \Sigma^{8}$.

Combining 3.1 with the Eells-Kuiper classification, we have the following examples:

COROllary 3.3. There is a smooth structure on a homotopy $\mathbf{H} P^{2}$ for which the only group $\mathbf{Z}_{p}$, $p$ an odd prime, which can act smoothly and quasi-linearly is $\mathbf{z}_{7}$.

There is a smooth structure on a 1-connected homology $\mathbf{H} P^{2}$ not of the homotopy type of $\mathbf{H} P^{2}$ for which the only group $\mathrm{Z}_{p}, p$ an odd prime, which can act smoothly and quasi-linearly is $\mathbf{Z}_{7}$.

Proof. Take $V_{h}$ for $h=49$ (recalling there are no quasi-linear $\mathbf{Z}_{3}$-actions) and $h=8$ respectively.

Observe that $Z_{3}$ has only one possible normal representation at each fixed-point component, so that every smooth $\mathbf{Z}_{3}$-action is quasi-linear. As we shall see below, every $\mathbf{Z}_{5}$ - or $\mathbf{Z}_{7}$-action on a homology $\mathbf{H} P^{2}$ must also be quasi-linear. While for $p=7$ the conditions of 3.1 do not yield any new restrictions to part d) of the Eells-Kuiper theorem, for $p=3$ or 5 we have

COROLlaRY 3.4. If $V$ is a smooth 1-connected homology $\mathbf{H} P^{2}$ with $p_{1}(V) Z$ \pm 2 (12), then $V$ admits no smooth $\mathbf{Z}_{3}$-action.

The manifolds $V_{h}$ for $h \equiv 8,56,113,161$ (168) all satisfy this condition.

If $V$ is a smooth 1-connected homology $\mathbf{H} P^{2}$ with $p_{1}(V) \neq \pm 2(20)$, then $V$ admits na smooth $\mathbf{Z}_{5}$-action.

The manifolds $V_{h}$ for

$$
h \equiv 8,49,57,64,112,113,168,169,217,224,232,273(280)
$$

satisfy this condition.

Suppose $\mathbf{Z}_{p}$ acts smoothly on $V$ with fixed points $p_{1}, p_{2}, p_{3}$. Let the normal representation at $p_{i}$ be $\left\{\theta_{i}^{j}(2 \pi / p)\right\}, j=1, \ldots, 4$. Then the Atiyah-Singer 
$G$-signature formula gives us the equation

$$
1=\sum_{i=1}^{3} \prod_{j=1}^{4} \cot \left(\theta_{i}^{j} \pi / p\right)
$$

The question then arises: Is there a collection $\left\{\theta_{i}^{j}\right\}, i=1, \ldots, 3, j=$ $1, \ldots, 4$, satisfying (*) which does not arise as the normal representations of a linear action?

A computer search has revealed that for $p=5$ or 7 there is not, so any $\mathbf{Z}_{5}$ or $\mathbf{Z}_{7}$-action on a homology $\mathbf{H} P^{2}$ must be quasi-linear. On the other hand, this search has found that for $p=11$, the collection $\{(1,2,3,4$,), $-(1,2,4,4),,(3,4,5,5)$,$\} , which cannot arise from a linear action (see$ paragraph 1), does satisfy (*).

We close with two problems:

Problem 1. Find all collections satisfying (*).

Problem 2. Which of these can arise as the normal representations of locally smooth actions?

\section{REFERENCES}

1. M. F. Atiyah and I. M. Singer, The index of elliptic operators.III, Ann. of Math. (2) 87 (1968), 546-604.

2. G. Bredon, Introduction to compact transformation groups, Academic Press, New York, 1972.

3. W. Browder, T. Petrie and C. T. C. Wall, The classification of free actions of cyclic groups of odd order on homotopy spheres, Bull. Amer. Math. Soc. 77 (1971), 455-459.

4. I. J. Dejter, Smooth $S^{1}$ manifolds in the homotopy type of $C P^{3}$, Michigan Math. J. 23 (1976), 83-95.

5. J. Eells and N. Kuiper, Manifolds which are like projective planes, Inst. Hautes Etudes Sci. Publ. Math. No. 14, 1962, pp. 5-46.

6. C. N. Lee, Cyclic group actions on homotopy spheres, (Proc. Conf. Transformation Groups, New Orleans, 1967), Springer, New York, 1968, p. 207.

7. S. Weintraub, Semi-free $\mathbf{Z}_{p}$-actions on highly-connected manifolds, Math. Z. 145 (1975), 163-185.

Department of Mathematics, Louisiana State University, Baton Rouge, lousiana 70803

Department of Mathematics, University of California, los Angeles, California 90024 